\title{
Co-rotating Circumstellar Clouds around Be Stars
}

\author{
L.A. Balona \\ South African Astronomical Observatory. P.O. Box 9, Observatory \\ 7935, Cape Town, South Africa
}

\begin{abstract}
A series of intensive high-resolution spectroscopic observations of 7 Be stars conducted over 5 years strongly indicates that the source of mass loss in these stars is to be found in magnetic activity coupled with rapid rotation. Magnetic centrifugal acceleration of ionized $\mathrm{H}$ and $\mathrm{He}$ along open field lines produce the circumstellar disk, while gas trapped in closed magnetic loops are responsible for the periodic variations.
\end{abstract}

\section{Introduction}

Many early-type Be stars are known to have photometric periods between 0.5 and $2.0 \mathrm{~d}$. The light curves are highly variable in shape and amplitude and can, at times, display a double-wave variation (Balona, Sterken \& Manfroid, 1991), but the period remains constant. There is no evidence for coherent multiperiodicity in the light curves. There is a correlation between the projected rotational velocity and the period showing that the photometric period is identical to the rotational period (Balona, 1995). The periodic line profile variations strongly resemble those of nonradial pulsations and in most cases can be modeled by a retrograde quadrupole mode (Rivinius et al., 2002). In order to study these variations, we undertook a series of intensive high-resolution spectroscopic observations (Balona, Aerts \& Štefl, 1999; Balona \& Kaye 1999, Balona, 1999 ; Balona \& Lawson 2001; Balona et al., 2001a; Balona et al., 2001b, Balona \& James 2002).

\section{Co-rotating Clouds}

The spectroscopic data show that the emission wings in the He lines (where they exist) vary in strength with the photometric period. This is direct evidence that the source of the periodic variation is to be found in the circumstellar material. In nearly all cases studied, two diametrically opposed clouds are present, which explains why the quadrupole mode is required in the pulsational interpretation. Further evidence for co-rotating clouds comes from the observation that the periodic line profile variations extend a little beyond the limit defined by the projected rotational velocity and that the periodic variations are strong in lines whose profiles are clearly distorted by circumstellar emission. In no case do we find evidence for multiperiodicity. Though the line variations may be complex, 
they repeat on a rotational cycle. Any analysis which assumes multiperiodicity will naturally give rise to multiperiodic components when fitting these data unless proper consideration is given to the underlying complexity.

It seems that the periodic variations could be interpreted as due to corotating clouds, implying the presence of a magnetic field. This suggests that the episodic outbursts which are characteristic of Be stars may be generated by release of magnetic energy similar to solar flares. We were able to observe a weak outburst in two stars, from which we concluded that these are localized events. Balona (1990) presents photometric evidence which also suggests that outbursts are localized and give rise to rotational modulation.

\section{Magnetic Centrifugal Acceleration}

The mechanism which drives the mass loss in Be stars has long remained a mystery. A crude calculation of various mass loss mechanisms shows that in the B-star range magnetic centrifugal acceleration is the most efficient mechanism (Strafella et al., 1998). Ionized gas expelled by a magnetic flaring event is forced into co-rotation by the magnetic field and accelerates along open field lines, forming an equatorial disk. Trapping of gas in closed magnetic loops at the intersections of the rotational and magnetic equators, forming to two co-rotating clouds, may be responsible for the periodic variability. The double-wave light curves may be easily understood in this way. Since the ionization fraction of helium drops rapidly towards the cooler B-type stars and since neutral helium is not affected by the magnetic field, the efficiency may be expected to drop towards later types. Hence one may expect the relative numbers of $\mathrm{Be}$ stars to drop towards late B. To compensate, late B-type stars would need to rotate closer to the critical velocity, as observed. This may also be the reason why the periodic variations are absent in late Be stars.

\section{References}

Balona, L.A., 1990, MNRAS 245, 92

Balona, L.A., 1995, MNRAS 277, 1547

Balona L.A., 1999, MNRAS 306, 407

Balona, L.A., Sterken, C., Manfroid, J., 1991, MNRAS 252, 93

Balona L.A., Kaye A.B., 1999, ApJ 521, 407

Balona L.A., Aerts C., Štefl S., 1999, MNRAS 305, 519

Balona L.A., James D.J., Lawson W.A., Shobbrook R.R., 2001a, MNRAS 324, 1041

Balona L.A., Handler G., Aerts C., Bozic H., Guinan E.F., James D.J., Kaye A.B., Shobbrook R.R., 2001b, MNRAS 327, 1288

Balona L.A., Lawson W.A., 2001, MNRAS 321, 131

Balona L.A., James D.J., 2002, MNRAS 332, 714

Rivinius Th., Baade D., Štefl S., Maintz M., 2002, in: C. Aerts, T.R. Bedding, J. Christensen-Dalsgaard (eds.), Radial and Nonradial Pulsations as Probes of Stellar Physics, ASP Conference Series 259, 240

Strafella F., Pezzuto S., Corciulo G.G., Bianchini A., Vittone A.A., 1998, ApJ 505, 299 